\title{
Investigating the impact of the HSE Healthier Vending Policy on unit sales of snack items
}

\author{
Marco McVey ${ }^{1}$, Isobel Stanley ${ }^{1}$, Agatha Lawless ${ }^{2}$, Sarah O’Brien ${ }^{2}$ and Celine Murrin ${ }^{1}$ \\ ${ }^{1} U C D$ School of Public Health, Physiotherapy and Sports Science, Dublin, Ireland and \\ ${ }^{2}$ Healthy Eating \& Active Living Programme, Health Service Executive, Dublin, Ireland
}

\section{Abstract}

Vending machines provide quick and easy access to snacks and, in general, provide few healthy options to consumers. Given this high availability of unhealthy foods in vending machines, consumers are likely to purchase and consume a less healthy option. The aim of this study was to investigate the impact of a Healthier Vending policy in all Health Service Executive (HSE) premises across the Republic of Ireland on unit sales of vending machine snacks. Under the policy, the contents of vending machines were altered so that healthier, Better Choice (BC), snacks would constitute a majority of the vending machine stock (minimum $60 \%$ ) in comparison to the less healthy, Other Choice (OC), snacks. Snacks were defined as BC if they contained: $\leq 150 \mathrm{kcals} / \mathrm{packet}, \leq 20 \mathrm{~g} / 100 \mathrm{~g}$ total fat,$\leq 5 \mathrm{~g} / 100 \mathrm{~g}$ saturated fat, $\leq 15 \mathrm{~g} / 100 \mathrm{~g}$ sugar, and $\leq 1.5 \mathrm{~g} / 100 \mathrm{~g}$ salt/sodium. Products stocked as OC should be $\leq 250 \mathrm{kcals} / \mathrm{packet}$. $\mathrm{BC}$ snacks were placed in prime slots and at eye-level in order to prompt the consumer towards buying a $\mathrm{BC}$ snack, with OC snacks being placed to incentivise the choice of BC snacks. Consumers were also incentivised to purchase BC snacks through written cues that appear on the vending machines. Sales data was provided by vending companies for 2015 (baseline) and 2016 (year 1). Unit sales of all snack types increased between the two time points, with the exception of dried fruit and nuts. The proportion of OC products purchased relative to total unit sales decreased following the intervention, as did the proportion of gum and mints and dried fruit and nuts sales. While the proportion of BC crisps purchased relative to total unit sales increased post-intervention, an increase in the proportion of OC bars purchased relative to total unit sales was also seen. Sales of both BC and OC dried fruit/nuts relative to total unit sales decreased. Our results indicate that policies aiming to improve the nutrition environment in health care settings, through increasing the availability of healthy products in vending machines and promoting their purchase, could be an effective method of reducing the amount of unhealthy products purchased overall. These policies can alter consumers' purchasing patterns and prompt consumers towards purchasing a healthier/unhealthier version of the same product, as seen with crisps and bars in this study.

\section{Conflict of Interest}

There is no conflict of interest. 